\title{
CAPACIDADE ABSORTIVA NO CONTEXTO DA INDÚSTRIA HOTELEIRA: UMA ANÁLISE DE PRÁTICAS DE ABSORÇÃO DE CONHECIMENTOS
}

\author{
Claudia Maria da Silva Bezerra \\ Doutoranda em Administração - UNINOVE \\ Universidade Nove de Julho \\ claudiamsbezerra@gmail.com \\ Heidy Rodriguez Ramos \\ Doutorado em Administração - FEA/USP \\ Universidade Nove de Julho \\ heidyrr@uni9.pro.br \\ Anderson Antônio de Lima \\ Doutorando em Administração - UNINOVE \\ Universidade Nove de Julho \\ andersonantoniodelima@yahoo.com.br \\ Eloisa Elena Shinohara \\ Doutoranda em Administração - UNINOVE \\ Universidade Nove de Julho \\ eloisa.elena@hotmail.com
}

\section{Resumo}

Objetivo do estudo: Identificar quais são as práticas existentes para captar e absorver conhecimentos externos em empreendimentos hoteleiros.

Metodologia/abordagem: $\mathrm{O}$ estudo foi desenvolvido com abordagem qualitativa, de caráter exploratório, por meio da análise de casos múltiplos de cinco hotéis, situados no Estado de São Paulo. A coleta de dados foi realizada com entrevistas semiestruturadas e a triangulação dos dados com sites e diários de bordo dos hotéis. A análise dos dados foi a partir da análise de conteúdo executada com o auxílio do Atlas.ti.

Originalidade/Relevância: As inovações resultantes da capacidade absortiva (ACAP), são fundamentais para a competitividade e sucesso de empresas de hotelaria, e são originadas por intensa interação entre funcionários e clientes envolvidos simultaneamente no processo de elaboração e aplicação das inovações.

Principais resultados: Os hotéis objetos deste estudo, possuem práticas de aquisição de conhecimentos externos similares, como participação em feiras e eventos do setor, análise das avaliações e sugestões dos hóspedes. A prática de aquisição predominante e compreendida como mais importante pelos respondentes são as melhorias geradas por meio da análise das avaliações e sugestões dos hóspedes indicadas nos guest coments (sugestões dos hóspedes) e nos meios digitais, redes sociais e OTA's (Agência de Reservas online).

Contribuições teóricas/metodológicas: O estudo está alinhado com a definição de Zahra e George (2002) para ACAP e contribui para a evolução epistemológica do campo de estudo da ACAP e compartilhamento de conhecimento, sobretudo, nos setores de turismo e hotelaria, preenchendo uma importante lacuna na literatura que apresenta escassez de estudos que investigaram as quatro dimensões referentes às práticas de ACAP Potencial (PACAP) e Realizada (RACAP), conforme apresentado por Zahra e George (2002).

Contribuições sociais/para a gestão: Como contribuição gerencial o estudo fornece um guia para os gestores das principais práticas identificadas e em como desenvolver estes processos de forma eficaz e consequentemente, promover inovações nos serviços prestados tendo em vista que os processos relacionados a ACAP são de extrema importância para a competitividade e para o desenvolvimento de inovações na indústria hoteleira.

Palavras-chave: Capacidade absortiva. Hotelaria. Turismo. Inovação. Vantagem competitiva sustentável.

\section{Cite como}

American Psychological Association (APA)

Bezerra, C. M. S., Ramos, H. R., Lima, A. A., \& Shinohara, E. E. (2022, jan./abr.). Capacidade absortiva no contexto da indústria hoteleira: uma análise de práticas de absorção de conhecimentos. PODIUM Sport, Leisure and Tourism Review, São Paulo, 11(1), 210-235. https://doi.org/10.5585/podium.v11i1.20310. 
Bezerra, C. M. S., Ramos, H. R., Lima, A. A., \& Shinohara, E. E. (2022, jan./abr.). Capacidade absortiva no contexto da indústria hoteleira: uma análise de práticas de absorção de conhecimentos

\title{
ABSORTIVE CAPACITY IN THE CONTEXT OF THE HOTEL INDUSTRY: AN ANALYSIS OF KNOWLEDGE ABSORPTION PRACTICES
}

\begin{abstract}
Study objective: To identify the existing practices to capture and absorb external knowledge in hotel projects. Methodology/approach: The study was developed with a qualitative, exploratory approach, through an analysis of multiple cases of five hotels, located in the State of São Paulo. Data collection was carried out with semistructured interviews and data triangulation with websites and hotel logbooks. Data analysis was based on content analysis and performed with the help of Atlas.ti.

Originality/Relevance: Innovations resulting from absorptive capacity are fundamental for the competitiveness and success of hotel companies, and are originated by intense interaction between employees and customers involved simultaneously in the process of elaboration and application of innovations.

Main results: The hotels object of this study have similar external knowledge acquisition practices, such as participation in fairs and events in the sector, analysis of reviews and suggestions from guests. The predominant acquisition practice and understood as the most important by respondents are the improvements generated through the analysis of reviews and suggestions from guests indicated in guest comments (guest suggestions) and in digital media, social networks and OTA's (Reservations Agency Online).

Theoretical/methodological contributions: The study is in line with the definition of Zahra and George (2002) for ACAP and contributes to the epistemological evolution of the field of study of absorptive capacity and knowledge sharing, especially in the tourism and hospitality sectors, filling an important There is a gap in the literature that shows a scarcity of studies that investigated the four dimensions referring to the practices of potential (PACAP) and realized (RACAP) absorptive capacities, as presented by Zahra and George (2002).

Social/Management Contributions: As a managerial contribution, the study provides a guide for managers on the main practices identified and on how to develop these processes effectively and consequently promote innovations in the services provided.
\end{abstract}

Keywords: Absorptive capacity. Hospitality. Tourism. Innovation. Sustainable competitive advantage.

\section{CAPACIDAD ABSORTIVA EN EL CONTEXTO DE LA INDUSTRIA HOTELERA: UN ANÁLISIS DE LAS PRÁCTICAS DE ABSORCIÓN DE CONOCIMIENTOS}

\section{Resumen}

Objetivo del estudio: Identificar las prácticas existentes para captar y absorber conocimiento externo en proyectos hoteleros.

Metodología/enfoque: El estudio se desarrolló con un enfoque cualitativo, exploratorio, mediante el análisis de múltiplos casos de cinco hoteles, situados en el Estado de São Paulo. La recolección de datos se llevó a cabo con entrevistas semiestructuradas y triangulación de datos con sitios web y diarios de los hoteles. El análisis de datos se basó en el análisis de contenido y se realizó con la ayuda de Atlas.ti.

Originalidad/Relevancia: Las innovaciones resultantes de la capacidad de absorción son fundamentales para la competitividad y el éxito de las empresas hoteleras, y se originan por una intensa interacción entre empleados y clientes involucrados simultáneamente en el proceso de elaboración y aplicación de las innovaciones.

Principales resultados: Los hoteles objeto de este estudio cuentan con prácticas similares de adquisición de conocimiento externo, tales como participación en ferias y eventos del sector, análisis de reseñas y sugerencias de los huéspedes. La práctica de adquisición predominante y entendida como la más importante por los entrevistados son las mejoras generadas a través del análisis de opiniones y sugerencias de los huéspedes, indicadas en los comentarios de los huéspedes y en los medios digitales, redes sociales y OTA's (Agencia de Reservas en línea).

Aportes teóricos/metodológicos: El estudio fue desarrollado de acuerdo con la definición de Zahra y George (2002) para ACAP y contribuye a la evolución epistemológica del campo de estudio de la capacidad de absorción y el intercambio de conocimientos, especialmente en los sectores del turismo y hotelería, llenando un vacío importante en la literatura que muestra una escasez de estudios que investigaron las cuatro dimensiones referentes a las prácticas de capacidades de absorción potencial (PACAP) y realizada (RACAP), tal como lo presentan Zahra y George (2002).

Contribuciones sociales/gerenciales: Como contribución gerencial, el estudio proporciona un guía para los gerentes sobre las principales prácticas identificadas y sobre cómo desarrollar estos procesos de manera efectiva $\mathrm{y}$ consecuentemente promover innovaciones en los servicios prestados.

Palabras clave: Capacidad absortiva. Hotelería. Turismo. Innovación. Ventaja competitiva sostenible. 
Bezerra, C. M. S., Ramos, H. R., Lima, A. A., \& Shinohara, E. E. (2022, jan./abr.). Capacidade absortiva no contexto da indústria hoteleira: uma análise de práticas de absorção de conhecimentos

\section{Introdução}

Capacidade absortiva (ACAP) é um constructo com diversas definições na literatura. Os conceitos existentes têm em comum o entendimento que trata da combinação de esforços das organizações com o objetivo de adquirir e utilizar conhecimentos externos para a obtenção de vantagem competitiva sustentável, ou seja, é um constructo multidimensional composto por processos de aquisição, assimilação, transformação e aplicação de conhecimentos (Cohen \& Levinthal, 1990; Lane et al., 2006; Lev et al., 2009; Zahra \& George, 2002; Zhang et al., 2019). As primeiras definições focaram no papel da área de Pesquisa e Desenvolvimento (P\&D) e posteriormente, as definições se expandiram para o nível organizacional e interorganizacional (Loureiro et al., 2019; Tsai, 2001; Zhang et al., 2019).

A literatura sobre capacidade absortiva e compartilhamento de conhecimentos cresceram rapidamente em diversas áreas do conhecimento (Enz \& Way, 2016). Contudo, este crescimento ainda é incipiente na indústria hoteleira (de Lima et al., 2021; Nieves \& DiazMeneses, 2017), apesar de ser uma indústria baseada em conhecimento (Enz \& Way, 2016; Hallin \& Marnburg, 2008; Noerchoidah \& Harjanti, 2019). De Lima et al. (2021) ao examinarem os estudos publicados sobre ACAP no contexto da indústria hoteleira constataram que apesar de existirem algumas publicações sobre compartilhamento de conhecimentos em empresas turísticas, estas publicações focaram em análises separadas das dimensões da ACAP, ou investigaram o impacto das fontes internas de conhecimento no desempenho da empresas (Binder, 2019; Bontis et al., 2015) ou no impacto de fontes externas de conhecimento (Hon \& Lui, 2016; Thomas \& Wood, 2015).

Além disso, Thomas e Wood (2015) destacam que empresas da indústria hoteleira são extremamente dependentes da busca por conhecimentos de fontes externas, uma vez que a incerteza no setor de turismo, a intensa concorrência entre empresas e as demandas dos clientes mudam constantemente e exigem inovações. Desta forma, as inovações que são resultantes da ACAP da organização possuem um papel fundamental para a competitividade e o sucesso de empresas ligadas ao setor da hospitalidade (Binder, 2019; Enz \& Way, 2016). As inovações nestas empresas frequentemente são originadas por meio de uma atividade humana intensa, na qual funcionários e clientes interagem e estão envolvidos simultaneamente no processo de elaboração e aplicação das inovações (Chang et al., 2018). Estudos sobre inovações na indústria hoteleira destacam o papel essencial dos funcionários que atuam em constante interação com 
os clientes (linha de frente) no desenvolvimento e aplicação de inovações (Cadwallader et al., 2010; Chang et al., 2011; Sainaghi et al., 2017).

O serviço prestado em hotéis geralmente demanda uma constante interação entre os funcionários e os clientes, além disso, a execução ou a entrega de um novo serviço ocorre de forma conjunta, desta forma, o cliente é um agente fundamental nas melhorias que são implementadas no serviço hoteleiro (Enz \& Way, 2016). As inovações são possíveis por meio de mudanças incrementais ou radicais em produtos ou serviços, neste sentido, as empresas hoteleiras necessitam constantemente promover melhorias em serviços levando em consideração dois fatores principais de inovação em serviços, o primeiro refere-se aos funcionários que interagem com os clientes e a disposição destes funcionários para promover melhorias nos serviços (Hon \& Lui, 2016), e o segundo está relacionado com a capacidade de absorção da organização para aquisição, assimilação, transformação e aplicação de conhecimentos (Cohen \& Levinthal, 1990; Lowik et al., 2012; Noerchoidah \& Harjanti, 2019).

As inovações na indústria hoteleira geralmente ocorrem por meio de inovações incrementais e normalmente são ocasionadas quando a organização enfrenta crises, como por exemplo, redução de faturamento e perda do mercado para concorrentes. Os conhecimentos que resultam em inovações incrementais são predominantemente captados no ambiente externo, seja por meio de hospedes, fornecedores, parceiros de negócios, colaboradores e concorrentes (Hon \& Lui, 2016), isto reforça a necessidade de investigar quais são as fontes de conhecimentos externos e como as organizações da indústria hoteleira assimilam, transformam e exploram os conhecimentos externos. Cabe lembrar que o setor de hospitalidade é intensivo em conhecimento como resultado da natureza dos serviços (Sainaghi et al., 2017). A prestação de serviços ocorre como resultado da interação entre clientes e funcionários, e é necessário que os funcionários tenham conhecimento das necessidades dos clientes (Chalkiti, 2012; Hallin \& Marnburg, 2008; Khale, 2002). Portanto, baseando-se nestes fatos, pode-se concluir que as empresas devem gerar constantemente novos conhecimentos para se manterem competitivas, sendo assim, os processos relacionados à capacidade de absorção são de extrema importância para a competitividade dos hotéis e para o desenvolvimento de inovações.

Devido à escassez na literatura de estudos sobre capacidade absortiva, mais especificamente de estudos que investiguem os processos de aquisição, assimilação, transformação e exploração de conhecimentos externos no contexto hoteleiro, o presente estudo busca identificar as práticas de aquisição, assimilação, transformação e aplicação de conhecimentos em hotéis (Enz \& Way, 2016). Esta pesquisa é relevante, uma vez que investiga 
práticas comuns, mas sobretudo, práticas diferentes, pois entende-se que cada empreendimento analisado está imerso em uma cultura organizacional única que influência nas práticas de capacidade de absorção adotadas. A questão de pesquisa que o estudo busca responder é quais são as práticas existentes para captar e absorver conhecimentos externos em empreendimentos hoteleiros? Neste sentido, o objetivo do estudo é identificar quais são as práticas existentes para captar e absorver conhecimentos externos em empreendimentos hoteleiros.

Para responder à questão de pesquisa, o estudo foi desenvolvido com abordagem qualitativa de caráter exploratório (Richardson, 2017; Vergara, 2013), por meio de análise de casos múltiplos (Bessant et al., 2010; Eisenhardt, 1989). A coleta de dados foi realizada por meio de entrevistas semiestruturadas com representantes de cinco hotéis situados no Estado de São Paulo, de diferentes portes e segmentos (Creswell, 2010; Deakin \& Wakefield, 2014). Também foi realizada uma coleta de dados secundários, utilizados na triangulação dos resultados. A análise dos dados foi a partir da análise de conteúdo e executada com o auxílio do Atlas.ti (Bardin, 2016; Flores, 1994).

O principal resultado deste estudo é que todos os hotéis pesquisados, mesmo que de forma indutiva, são capazes de empregar as dimensões do modelo de Zahra e George (2002), a partir da aquisição, assimilação, transformação e aplicação de conhecimentos. Os hotéis analisados possuem práticas de aquisição de conhecimentos externos similares, como por exemplo, participação em feiras e eventos do setor, análise das avaliações e sugestões dos hóspedes. A prática de aquisição predominante e compreendida como mais importante pelos respondentes são as melhorias geradas por meio da análise das avaliações e sugestões dos hóspedes, indicadas nos guest coments (sugestões dos hóspedes) e nos meios digitais, redes sociais e OTA’s (Agência de Reservas on-line).

$\mathrm{O}$ estudo está estruturado em cinco seções. $\mathrm{Na}$ introdução se apresenta uma breve contextualização sobre capacidade absortiva, a relevância do estudo, a questão de pesquisa e seus objetivos. Na seção seguinte é apresentada a fundamentação teórica em que são discutidos o surgimento, evolução e as principais correntes teóricas sobre capacidade absortiva presentes na literatura. A terceira seção apresenta o método adotado no estudo, a abordagem metodológica, os instrumentos de coleta de dados e as ferramentas para a análise de dados. $\mathrm{Na}$ seção seguinte se realiza a análise e discussão dos dados coligidos. Por fim, as considerações finais do estudo são apresentadas, com as contribuições gerenciais e teóricas, assim como as limitações e sugestões de estudos futuros. 


\section{Fundamentação teórica}

Capacidade absortiva

O termo capacidade absortiva (ACAP) foi introduzido por Cohen e Levinthal (1990). Os autores definiram ACAP como uma capacidade da organização em adquirir, valorizar, assimilar e gerar novos conhecimentos com o objetivo de obter vantagem competitiva sustentável (Dzhengiz \& Niesten, 2019; Gebauer et al., 2012; Lev et al., 2009; Zahra \& George, 2002). A capacidade absortiva em outros estudos foi definida como um conjunto amplo de habilidades essenciais para codificar o conhecimento tácito em explícito para que a organização consiga utilizar e remodelar de acordo com as condições ambientais (Mowery \& Oxley, 1995; Zahra \& George, 2002). O conhecimento tácito é o conhecimento internalizado que está no subconsciente dos indivíduos, este conhecimento não é verbalizado ou transferido com facilidade, por outro lado, o conhecimento explícito é verbalizado e externalizado, trata-se das habilidades dos indivíduos (Nonaka \& Takeuchi, 1997; Polanyi, 1966; Tatto \& Bordin, 2016).

Apesar de não ser um constructo com definição consolidada na literatura, um corpo expressivo de estudiosos da temática entendem que a capacidade absortiva é um tipo específico de capacidade dinâmica, sendo que, o conceito de capacidade absortiva está inserido em estudos que pesquisaram as capacidades dinâmicas (Teece, 2007; Zollo \& Winter, 2002). As capacidades dinâmicas podem ser definidas como a capacidade gerencial de recombinar e reconfigurar os recursos e as rotinas de uma organização de acordo com as pressões e mudanças ambientais, são benéficas para a organização, sobretudo, em mercados dinâmicos e turbulentos (Gebauer et al., 2012; Pisano, 2017; Teece, 2007; Zahra et al., 2006).

O estudo mais influente que compreende a capacidade absortiva como um tipo específico de capacidade dinâmica foi apresentado por Zahra e George (2002). Estes autores apresentaram um modelo que divide a capacidade absortiva em capacidades potenciais (PACAP), que estão ligadas aos processos de aquisição de conhecimentos externos e assimilação do conhecimento pelos membros da organização e capacidades realizadas (RACAP), que estão ligadas à transformação do conhecimento adquirido e assimilado para a organização conseguir utilizar e aplicar tal conhecimento, seja na forma de melhorias ou na criação de novos produtos, serviços e processos (Gebauer et al., 2012). As duas dimensões (PACAP e RACAP), introduzidas na literatura por Zahra e George (2002), são apresentadas na Figura 1, adaptada dos próprios autores, em que são comparados seus componentes, papel e importância. 
Bezerra, C. M. S., Ramos, H. R., Lima, A. A., \& Shinohara, E. E. (2022, jan./abr.). Capacidade absortiva no contexto da indústria hoteleira: uma análise de práticas de absorção de conhecimentos

Figura 1.

Dimensões da Capacidade Absortiva

\begin{tabular}{|c|c|c|c|}
\hline Dimensões & Componentes & Papel e Importância & Citações \\
\hline Aquisição & $\begin{array}{c}\text { - Investimentos prévios } \\
\text { - Conhecimento prévio } \\
\text { - Intensidade } \\
\text { - Velocidade } \\
\text { - Direção }\end{array}$ & $\begin{array}{c}\text { - Busca de escopo } \\
\text { - Esquema perceptivo } \\
\text { - Novas conexões } \\
\text { - Aprendizado rápido } \\
\text { - Aprendizado de qualidade }\end{array}$ & $\begin{array}{l}\text { Boynton, Zmud, \& Jacobs (1994); } \\
\text { Cohen \& Levinthal (1990); Keller } \\
\text { (1996); Kim (1998); Lyles \& } \\
\text { Schwenk (1992); Mowery, Oxley, } \\
\text { \& Silverman (1996); Van Wijk, } \\
\text { Van den Bosch, \& Volberda } \\
\text { (2001); Veugelers (1997) }\end{array}$ \\
\hline Assimilação & Compreensão & $\begin{array}{l}\text { - Interpretação } \\
\text { - Compreensão } \\
\text { - Aprendizado }\end{array}$ & $\begin{array}{c}\text { Dodgson (1993); Fichman \& } \\
\text { Kemerer (1999): Kim (1998); Lane } \\
\text { \& Lubatkin (1998); Szulanski } \\
\text { (1996) }\end{array}$ \\
\hline Transformação & $\begin{array}{l}\text { - Internalização } \\
\text { • Conversão }\end{array}$ & $\begin{array}{l}\text { • Sinergia } \\
\text { • Recodificação } \\
\text { • Bissociação }\end{array}$ & $\begin{array}{c}\text { Fichman \& Kemerer (1999); } \\
\text { Koestler (1966); Kim (1997b, } \\
\text { 1998); Smith \& DeGregorio (em } \\
\text { imprensa) }\end{array}$ \\
\hline Exploração & $\begin{array}{l}\text { •Uso } \\
\cdot \text { Implementação }\end{array}$ & $\begin{array}{l}\text { - Competências principais } \\
\text { - Captação de recursos }\end{array}$ & $\begin{array}{c}\text { Cohen \& Levinthal (1990); } \\
\text { Dodgson (1993); Kim (1998); Lane } \\
\text { \& Lubatkin(1998); Szulanski } \\
\text { (1996); Van den Bosch, Volberda, } \\
\text { \& de Boer (1999); Van Wijk, Van } \\
\text { den Bosch, \& Volberda (2001) }\end{array}$ \\
\hline
\end{tabular}

Fonte: Adaptado de Zahra e George (2002).

As experiências anteriores das organizações são antecedentes da capacidade absortiva, estas experiências definem o lócus de pesquisa tecnológica de uma organização, frequentemente as organizações buscam informações em áreas onde obtiveram sucesso (Christensen, 1997; Cyert \& March, 1963). A experiência também é relacionada à memória organizacional (Walsh \& Ungson, 1991) e trata-se do depósito do conhecimento de uma organização (Herriot et al., 1985; Moorman \& Miner, 1998; Zahra \& George, 2002).

A capacidade absortiva potencial e a realizada são afetadas pelos mecanismos de integração social que agem para minimizar a lacuna entre capacidade potencial e realizada, ampliando a eficiência da organização nos processos de aquisição, assimilação, transformação e aplicação de conhecimentos, uma vez que facilitam o compartilhamento de conhecimentos dentro das organizações, por exemplo, metodologia de resolução de problemas, rotação de cargos, círculos de qualidade (Zahra \& George, 2002; Vega-Jurado et al., 2008).

Os gatilhos de ativação são compreendidos como eventos que motivam ou obrigam que as organizações respondam às pressões, oscilações e mudanças no ambiente externo. Os gatilhos de ativação podem ser crises organizacionais, como desempenho abaixo do esperado, ou eventos importantes que são capazes de redesenhar a estratégia de uma empresa (por 
exemplo, fusões e lançamento de um produto inovador por um concorrente), uma crise, apesar de negativa, possibilita que a organização amplie os esforços para alcançar e aprender novas habilidades e desenvolver novos conhecimentos que aumentem a capacidade absortiva (Salunke et al., 2019; Winter, 2000; Zahra \& George, 2002).

Uma definição de capacidades dinâmicas que enfatiza a importância das rotinas, afirma que trata-se de padrões sistemáticos inseridos nas operações das organizações que estão relacionados a criação e a adaptação de rotinas operacionais, as rotinas mudam de acordo com mecanismos de aprendizado (Parmigiani \& Howard-Grenville, 2011; Zollo \& Winter, 2002). Os processos que compõem a capacidade absortiva de aquisição, assimilação, transformação e aplicação de conhecimentos internos e externos podem ser definidos como rotinas (Flor et al., 2018; Lewin et al., 2011). As rotinas com padrões regulares e previsíveis das organizações são como genes biológicos, ou seja, são herdáveis e selecionáveis pelo ambiente, sendo assim, fornecem a capacidade de mudança evolutiva para as organizações (Nelson \& Winter 2009; Parmigiani \& Howard-Grenville, 2011), especialmente em eventos ou situações adversas (Salunke et al., 2019; Zahra \& George, 2002).

As rotinas também podem ser entendidas como práticas administrativas ou organizacionais que estão interligadas nas tarefas diárias de acordo com costumes compartilhados pela cultura organizacional (Simcsik, 2001). Gebauer et al., (2012) em seu estudo, analisaram as rotinas e destacaram que as práticas de identificação de novos conhecimentos oriundos de diversas fontes externas, como por exemplo, as práticas de geração de informações sobre o ambiente de negócios relevantes para novas oportunidades de negócios, práticas de aquisição, seleção e retenção de documentos captados do ambiente externo, foram identificadas como indicadores de processos de aquisição, assimilação, transformação e aplicação de conhecimentos, descritas na Figura 2. 
Bezerra, C. M. S., Ramos, H. R., Lima, A. A., \& Shinohara, E. E. (2022, jan./abr.). Capacidade absortiva no contexto da indústria hoteleira: uma análise de práticas de absorção de conhecimentos

\section{Figura 2.}

Práticas Relacionadas às Duas Dimensões de Capacidade Absortiva

\begin{tabular}{|c|c|}
\hline Processos de Conhecimentos & Indicadores e noções referentes aos construtos \\
\hline \multicolumn{2}{|c|}{ Capacidade Absortiva Potencial - PACAP } \\
\hline $\begin{array}{l}\text { Processos de aprendizagem Processo exploratório de } \\
\text { aprendizagem }\end{array}$ & $\begin{array}{l}\text { - Abertura para fontes de conhecimento externos } \\
\text { - Reconhecimento de fontes de conhecimento externas } \\
\text { - Engajamento em projetos conjuntos de criação de conhecimento } \\
\text { - Regularidade das reuniões com externos } \\
\text { - Motivação para usar fontes de conhecimento externas } \\
\text { - Identificação de novos conhecimentos em fontes externas } \\
\text { - Gerar informações sobre o ambiente de negócios relevantes para novas } \\
\text { oportunidades de negócios } \\
\text { - Aquisição de conhecimento através de diversas fontes } \\
\text { - Seleção e retenção de conhecimentos obtidos a partir de fontes externas } \\
\text { - Classificar e internalizar o conhecimento adquirido }\end{array}$ \\
\hline Processos de aprendizagem assimilativos & $\begin{array}{l}\text { - Interpretação compartilhada do conhecimento recém-adquirido } \\
\text { - Discussão do conhecimento adquirido } \\
\text { - Obtendo a compreensão coletiva do conhecimento adquirido } \\
\text { - Integração de novos conhecimentos na base de conhecimento da empresa } \\
\text { - Disseminação de novos conhecimentos em toda a empresa } \\
\text { — Utilização de ferramentas para disseminar conhecimento em toda a empresa }\end{array}$ \\
\hline \multicolumn{2}{|c|}{ Capacidade Absortiva Realizada - RACAP } \\
\hline Processos transformadores de aprendizagem & $\begin{array}{l}\text { - Manutenção e reativação do conhecimento } \\
\text { - Criação de novos conhecimentos a partir do conhecimento adquirido } \\
\text { - Reconstruindo o conhecimento adquirido } \\
\text { - Facilitando a transferência e novas associações relativas ao conhecimento } \\
\text { - Interpretação discursiva do conhecimento } \\
\text { - Agregando novos conhecimentos ao conhecimento adquirido } \\
\text { - Combinação construtiva e recombinação de conhecimento } \\
\text { - Vinculando o conhecimento existente com novos insights }\end{array}$ \\
\hline
\end{tabular}

Fonte: Adaptado de Gebauer et al. (2012).

\section{Capacidades absortivas na indústria hoteleira}

Apenas alguns estudos sobre capacidade absortiva no campo da indústria hoteleira são apresentados na literatura (de Lima et al., 2021). Dentre os estudos neste campo, Binder (2019) analisou separadamente o impacto das fontes internas de captação de conhecimentos no desempenho organizacional e Hon e Lui (2016) analisaram a influência das fontes externas de conhecimento na performance organizacional.

A indústria hoteleira tem a peculiaridade de ser caracterizada pela utilização constante de novos conhecimentos, devendo captar e processar conhecimentos externos para implantar melhorias nos serviços prestados (de Lima et al., 2021). Uma parte destas informações são captadas por meio da interação com os hóspedes, que possuem expectativas sobre o serviço, comparam com experiências anteriores e sugerem melhorias (de Lima et al., 2021). Esta 
interação ocorre por meio de um processo extensivo e constante de produção de compartilhamento de conhecimentos, que é compreendido pela ótica da capacidade absortiva (Hallin \& Marnburg, 2008; Noerchoidah \& Harjanti, 2019).

A inovação é o resultado deste processo eficaz de absorção de conhecimentos, incremental ou disruptiva, que impacta positivamente na competitividade e no sucesso dos empreendimentos hoteleiros (Binder, 2019; Enz \& Way, 2016). Colaboradores e hóspedes estão concentrados simultaneamente no processo de criação e execução das inovações, que ocorre por meio do relacionamento humano e intensivo entre ambos (Cadwallader et al., 2010; Chang et al., 2011; Chang et al., 2018). Dentro deste contexto, o papel dos colaboradores, principalmente os da linha de frente, é essencial para os processos de aquisição, assimilação, transformação e aplicação dos conhecimentos externos, que devem ser priorizados pela organização para que aconteçam de forma eficaz, definindo, assim, a capacidade de absorção de conhecimentos externos pela organização (Cohen \& Levithal, 1990; Lowik et al., 2012; Noerchoidah \& Harjanti, 2019).

A indústria hoteleira é afetada por rápidas mudanças, principalmente no ambiente externo, que podem se tornar um obstáculo para a criação e manutenção das vantagens competitivas (Wordl Travel \& Tourism Council, 2018). A gestão do conhecimento pode promover inovações e mudanças no desempenho organizacional (por exemplo, novos serviços) que darão suporte para a superação de obstáculos, desempenhando um papel fundamental no sucesso da organização (Kim \& Lee, 2012; Pattanasing et al., 2019).

O presente estudo está alinhado com a definição de Zahra e George (2002) que entende que a capacidade absortiva é um tipo específico de capacidades dinâmicas, que está relacionada à capacidade da empresa em identificar, assimilar, transformar e aplicar conhecimentos com o objetivo de adquirir vantagem competitiva de forma sustentada e que pode ser reconfigurada de acordo com as pressões, oscilações e mudanças no ambiente. O estudo também assume que os recursos são formados geralmente por combinações de rotinas, por sua vez, a combinação e reconfiguração de recursos geram capacidades dinâmicas, sendo assim, as rotinas são fundamentais nas capacidades dinâmicas (Parmigiani \& Howard-Grenville, 2011). A capacidade absortiva pode ser compreendida como um tipo específico de capacidade dinâmica da organização (Zahra \& George, 2002), a codificação realizada por Gebauer et.al, 2012 de práticas que podem identificar rotinas de aquisição, assimilação, transformação e aplicação de conhecimentos. Este estudo utilizará estas abordagens para analisar quais são as práticas de captação e absorção de conhecimentos externos em empreendimentos hoteleiros. 
Bezerra, C. M. S., Ramos, H. R., Lima, A. A., \& Shinohara, E. E. (2022, jan./abr.). Capacidade absortiva no contexto da indústria hoteleira: uma análise de práticas de absorção de conhecimentos

\section{Procedimentos metodológicos}

O estudo foi desenvolvido com abordagem qualitativa de corte transversal. A pesquisa qualitativa é a mais adequada para os estudos que procuram descrever a complexidade de determinado problema, analisar a interação de certas variáveis, compreender e classificar processos dinâmicos (Richardson, 2017). A pesquisa de caráter exploratório é realizada em áreas na qual há pouco conhecimento acumulado, tem uma natureza de sondagem, não comporta hipóteses, mas estas podem surgir durante ou ao final da pesquisa (Vergara, 2013).

O estudo de caso é uma estratégia de pesquisa que foca o entendimento de um presente dinâmico, que tem um conjunto de singularidades (Eisenhardt, 1989). O uso de estudo de casos múltiplos permite comparar empresas de uma mesma cadeia de suprimentos de acordo com o mesmo protocolo de estudo de caso, além de confrontar e comparar os casos e produzir resultados mais confiáveis e generalizáveis (Benbasat et al., 1987). A opção pelo estudo de casos múltiplos se justifica porque os limites do fenômeno não estão claramente definidos no início da pesquisa. Esta modalidade de estudo examina, também, o fenômeno no seu ambiente natural, utilizando múltiplos métodos de coleta de dados para reunir informações sobre uma ou mais variáveis (pessoas, grupos ou organizações) (Bessant et al., 2010). A Figura 3 exemplifica a metodologia abordada neste estudo.

\section{Figura 3.}

Metodologia de Pesquisa

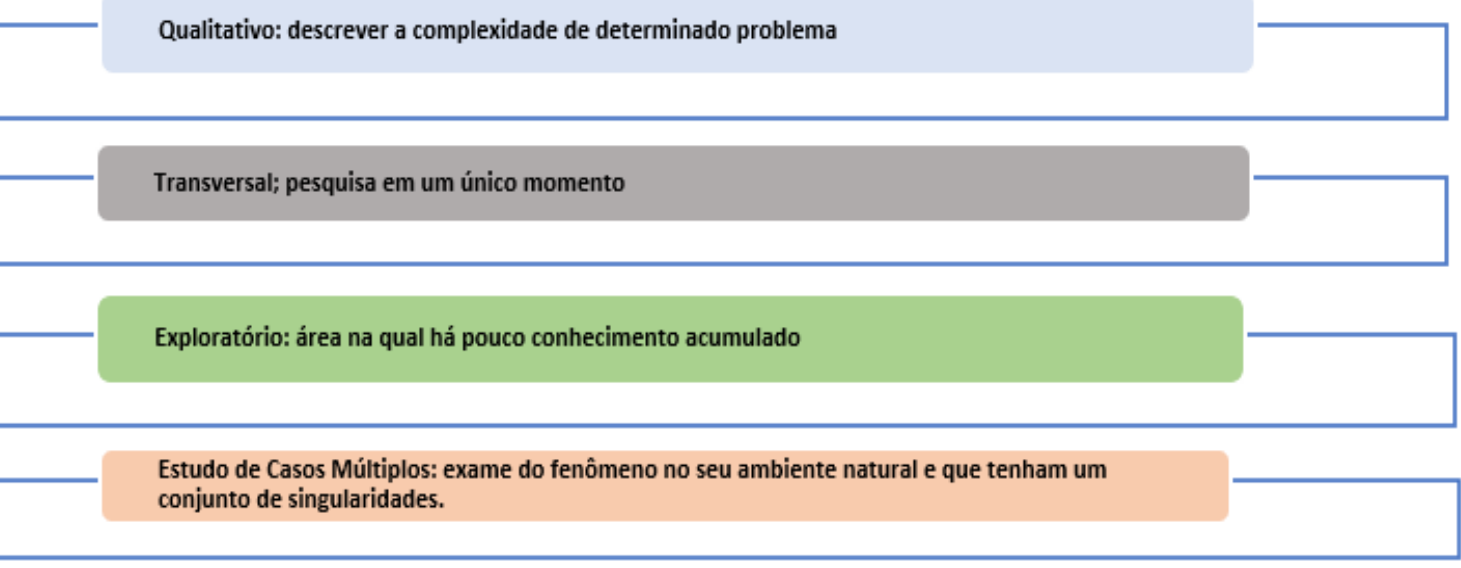

Fonte: Os autores. 
Neste estudo, a amostra foi composta por gerentes de cinco hotéis distintos, situados no Estado de São Paulo. Foram selecionados respondentes com experiência no ramo de hotelaria. A Tabela 1 descreve o perfil dos entrevistados com as características socioculturais.

Tabela 1.

Perfil dos Respondentes

\begin{tabular}{|c|c|c|c|c|c|}
\hline Entrevistado & Cargo & $\begin{array}{l}\text { Tempo de } \\
\text { Empresa }\end{array}$ & Grau de Instrução & Idade & Sexo \\
\hline EH-1 & Gerente RH & 9 anos & Superior em Recursos Humanos & 36 & Masculino \\
\hline EH-2 & Gerente Geral & 2 anos & $\begin{array}{l}\text { Superior em Administração de } \\
\text { Empresas e Economia }\end{array}$ & 57 & Masculino \\
\hline EH-3 & Gerente Geral & 10 anos & $\begin{array}{l}\text { Superior em Administração de } \\
\text { Empresas }\end{array}$ & 35 & Masculino \\
\hline EH-4 & Gerente Geral & 5 anos & Superior em Hotelaria & 42 & Masculino \\
\hline EH-5 & Gerente Geral & 7 anos & $\begin{array}{l}\text { Superior em Administração de } \\
\text { Empresas }\end{array}$ & 38 & Masculino \\
\hline
\end{tabular}

Fonte: Os autores.

A Tabela 2 apresenta a descrição do padrão dos hotéis em que os entrevistados trabalham. Todos os hotéis estão situados no estado de São Paulo na região da Grande São Paulo.

Tabela 2.

Padrão dos Hotéis

\begin{tabular}{|c|c|c|c|c|c|}
\hline Entrevistado & Característica & $\mathbf{N}^{0}$ Quartos & $\begin{array}{c}\mathbf{N}^{\mathbf{o}} \\
\text { Funcionários }\end{array}$ & $\begin{array}{l}\text { Idade do } \\
\text { Hotel }\end{array}$ & Tipo do Hotel \\
\hline EH-1 & $\begin{array}{l}\text { Médio } \\
\text { Porte }\end{array}$ & 216 & 89 & anos & Executivo \\
\hline EH-2 & $\begin{array}{l}\text { Pequeno } \\
\text { Porte }\end{array}$ & 25 & 28 & anos 40 & $\begin{array}{l}\text { Casamentos, } \\
\text { Eventos e Lazer }\end{array}$ \\
\hline EH-3 & $\begin{array}{l}\text { Pequeno } \\
\text { Porte }\end{array}$ & 57 & 62 & $\operatorname{anos}^{37}$ & Lazer \\
\hline EH-4 & $\begin{array}{l}\text { Médio } \\
\text { Porte }\end{array}$ & 108 & 65 & anos & Lazer \\
\hline EH-5 & $\begin{array}{l}\text { Pequeno } \\
\text { Porte }\end{array}$ & 48 & 32 & anos & Lazer \\
\hline
\end{tabular}

Fonte: Os autores.

A coleta de dados foi realizada por meio de entrevistas com roteiro semiestruturado (Tabela 3), validado por dois pesquisadores especialistas em ACAP, Turismo e Hospitalidade. Um pré-teste foi realizado com o entrevistado EH-1 e o roteiro foi ajustado, a fim de incluir as inovações adotadas em virtude da pandemia da Covid-19. As entrevistas foram realizadas por meio eletrônico (Hangouts e Skype), no mês de julho de 2020, em meio a pandemia da Covid- 
Bezerra, C. M. S., Ramos, H. R., Lima, A. A., \& Shinohara, E. E. (2022, jan./abr.). Capacidade absortiva no contexto da indústria hoteleira: uma análise de práticas de absorção de conhecimentos

19 e em um período de isolamento social. As entrevistas on-line permitem flexibilidade dos participantes e as tecnologias utilizadas são práticas, fáceis de usar e econômicas (Deakin \& Wakefield, 2014). A entrevista é um método popular e comumente utilizado em pesquisas nas ciências sociais, pois os participantes podem fornecer informações históricas importantes, além de um panorama que mostra diversas perspectivas, que possibilita o surgimento de dados que não foram antecipados no desenho da pesquisa ou sugeridos pela literatura (Creswell, 2010).

Tabela 3.

Roteiro da Entrevista

Roteiro de Entrevista Semiestruturado - Capacidade Absortiva em Hotéis

\begin{tabular}{c|c|}
\multicolumn{2}{c|}{ Roteiro de Entrevista Semiestruturado - Capacidade Absortiva em Hotéis } \\
\hline Questões Sociodemográficas & Questões Relacionadas às Características da Organização \\
\hline Qual é o cargo que ocupa na empresa? & Qual é o tipo do hotel (executivo, lazer ou convenções)? \\
\hline Quanto tempo você tem de trabalho aqui? & Qual é o porte do Hotel (pequeno, médio ou grande)? \\
\hline Quais foram suas experiências anteriores? & Qual é a quantidade de quartos? \\
\hline Qual é o seu grau de instrução? & Qual é o número de funcionários? \\
\hline Qual é sua idade? & Qual é a idade do empreendimento? \\
\hline \multicolumn{2}{c}{ Questões relacionadas a ACAP (PACAP E RACAP) }
\end{tabular}

Questões relacionadas a ACAP (PACAP E RACAP)

Descreva três ações voltadas para a inovação que o hotel adotou nos últimos dois anos.

Onde buscam informações e conhecimentos para promover melhorias ou inovações nos serviços prestados?

Comente sobre reuniões com fornecedores, parceiros, clientes ou terceirizados sobre possíveis melhorias na estrutura, processos e serviços prestados pelo hotel.

Como é realizada a seleção e armazenamento das informações e conhecimentos obtidos por meio de fontes externas?

Descreva a forma que os resultados das pesquisas de satisfação com os clientes são discutidos com funcionários.

De qual forma a organização incentiva os funcionários para compartilhar conhecimentos entre eles?

Cite um exemplo de conhecimento externo que foi transformado pela organização e aplicado para melhorar um processo ou serviço?

Quais os incentivos para os colaboradores se reciclarem? O hotel apoia a participação dos colaboradores ou fornece algum subsídio para atualizações?

Relate três práticas existentes em departamentos do hotel que foram adaptadas de conhecimentos adquiridos por meio de fontes externas?

Descreva como ocorrem os encontros periódicos entre áreas ou departamentos para compartilhamento de conhecimentos e resolução de problemas que afetam a organização.

Fonte: Desenvolvido pelos autores

Com a autorização dos respondentes, as entrevistas foram gravadas e transcritas. $\mathrm{O}$ tempo médio de cada entrevista foi de 40 a 50 minutos. A transcrição foi realizada logo após cada entrevista resultando em 39 páginas. Um e-mail destas transcrições foi encaminhado a cada participante, como critério de validade e confiabilidade (Golafshani, 2003). Após todos os participantes validarem o conteúdo das transcrições, o material foi analisado para verificar convergências dos dados coligidos. A técnica de análise de dados foi a análise de conteúdo para 
identificar características, categorias, esquemas e modelos similares nos discursos dos respondentes (Bardin, 2016; Flores, 1994).

A fim de conferir validade, fidedignidade, bem como credibilidade ao estudo, foi adotada a estratégia de triangulação das fontes de evidências (Eisenhardt, 1989; Flick, 2020). Os dados coletados nas entrevistas, bem como os dados fornecidos nos diários de campo e portfólios de sugestões de clientes fornecidos pelos respectivos hotéis pesquisados, foram imputados no software Atlas $t i$ (Woods et al., 2016) para que esses dados pudessem durante a análise de conteúdo, ser confrontados com a literatura revisada.

A priori foi realizada uma codificação do conteúdo e iniciou-se a etapa exploratória. Com a ajuda do Atlas.ti, foram classificados os aspectos convergentes, os dados brutos foram transformados em uma representação do conteúdo e novos códigos foram criados a partir desta etapa. A próxima etapa foi a interpretação dos dados, que exige atenção dos pesquisadores em comparar os achados da classificação do software com as categorias criadas pela teoria. Seguindo os critérios recomendados por Bardin (2016) e Flores (1994), as falas dos entrevistados e a documentação fornecida foram confrontadas com as categorias e os códigos gerados a partir da literatura, de modo a ilustrar ou representar a essência do fenômeno (Tuzzo \& Braga, 2016).

Ao analisar as narrativas foram destacados os casos que convergiam para o modelo de Zahra e George (2002) e Gebauer et al. (2012). Foram criados os seguintes códigos a partir destas narrativas e imputados no Atlas.ti para a análise de conteúdo: Aplicação/Exploração, Aquisição, Assimilação, Disseminação de Informações, Inovação e Transformação.

Com o auxílio do Atlas ti foi possível estabelecer nos dados analisados relações com as categorias estabelecidas de acordo com a revisão da literatura e assim executar a análise indutiva de conteúdo das entrevistas e dos documentos fornecidos (Woods et al., 2016). Esperase, com isso, poder contribuir com o aprimoramento e ampliação epistemológica do campo de estudo da capacidade absortiva, identificando as práticas de absorção de conhecimento sobretudo, no contexto da hotelaria.

\section{Análise e discussão dos resultados}

A amostra que compôs este estudo foi formada por gerentes de cinco hotéis distintos, situados no Estado de São Paulo. Os respondentes possuíam de 2 a 10 anos de tempo de empresa, todos tinham nível superior de instrução e eram do sexo masculino. Em relação aos 
Bezerra, C. M. S., Ramos, H. R., Lima, A. A., \& Shinohara, E. E. (2022, jan./abr.). Capacidade absortiva no contexto da indústria hoteleira: uma análise de práticas de absorção de conhecimentos

hotéis que compuseram a amostra, três eram de pequeno porte e possuíam de 25 a 57 funcionários e dois eram de médio porte com 65 e 89 funcionários. Com base nas respostas dos entrevistados e na literatura consultada, a Tabela 4 apresenta a descrição dos códigos gerados, bem como as principais descobertas relacionadas ao tema resultantes da pesquisa.

Tabela 4.

Descrição de Códigos e Principais Descobertas

\begin{tabular}{c|c|c}
\hline & Código & Principais Descobertas \\
\hline$\circ$ & Aplicação/Exploração & Implantação de novo projeto \\
\hline$\circ$ & Aquisição & Feiras e eventos, Pesquisa de avaliação de hospedagem, Consultorias \\
\hline$\circ$ & Assimilação & Edição de manuais, Loog Book (livro de registro de cada setor) \\
\hline$\circ$ & Disseminação de Informações & Reuniões regulares \\
\hline$\circ$ & Inovação & Finalização de projeto diferenciado \\
\hline$\circ$ & Transformação & Análise de mercado para adaptação e implantação de novo serviço ou \\
produto
\end{tabular}

Fonte: Os autores (Atlas.ti).

A análise realizada com o auxílio do software Atlas.ti gerou um índice de magnitude dos códigos, ou seja, com os códigos que mais foram citados nas narrativas dos respondentes. A Aquisição (75) e a Inovação (59) foram os temas que emergiram como sendo os mais importantes na percepção dos entrevistados, seguidos da assimilação (51) e disseminação de informações (51), conforme apresentado na Tabela 5.

Tabela 5.

Índice de Magnitude

\begin{tabular}{|c|c|c|c|c|c|c|c|c|}
\hline & EH-1 & EH-2 & EH-3 & EH-4 & EH-5 & Documentos & $\begin{array}{c}\text { Referencial } \\
\text { Teórico }\end{array}$ & $\begin{array}{c}\text { Magnitude } \\
\text { Total }\end{array}$ \\
\hline Aquisição & 9 & 19 & 7 & 8 & 10 & 10 & 12 & 75 \\
\hline Assimilação & 8 & 13 & 2 & 5 & 9 & 8 & 6 & 51 \\
\hline $\begin{array}{l}\text { Disseminação de } \\
\text { informações }\end{array}$ & 6 & 10 & 3 & 7 & 7 & 5 & 13 & 51 \\
\hline Transformação & 8 & 12 & 0 & 5 & 5 & 6 & 7 & 43 \\
\hline Novas Práticas & 2 & 11 & 2 & 8 & 8 & 7 & 10 & 48 \\
\hline $\begin{array}{l}\text { Aplicação/ } \\
\text { Exploração }\end{array}$ & 1 & 5 & 3 & 2 & 2 & 3 & 11 & 27 \\
\hline Inovação & 6 & 20 & 1 & 10 & 8 & 9 & 5 & 59 \\
\hline Totais & 40 & 90 & 18 & 45 & 49 & 48 & 64 & 354 \\
\hline
\end{tabular}


A literatura sugere que a indústria hoteleira é baseada em conhecimento e que esse processo se dá especialmente pela interação com os hóspedes (Enz \& Way, 2016; Hallin \& Marnburg, 2008; Noerchoidah \& Harjanti, 2019). Este estudo inferiu que as principais práticas de captação e absorção de conhecimentos externos corroboram com os indicadores na dimensão de Aquisição de Gebauer et al. (2012). Nos empreendimentos analisados, estas práticas se dão, com maior regularidade, pela interação entre hóspedes e funcionários, por meio dos guest coments (sugestões dos hóspedes) e pelas avaliações realizadas nos meios digitais, redes sociais e OTA's (Agência de Reservas on-line). Essas interações permitem extensiva transferência e troca de conhecimentos, conforme relato de alguns entrevistados:

"Tem todos estes canais de comunicação com o hóspede, né? [...] te avalia no Trip, com a avaliação dá para saber a impressão do hóspede em relação ao trabalho que a gente está executando, né? E dessas ideias vão surgindo aqui novas ideias em torno de melhoria de processo." (EH-2)

"[...] o principal instrumento que utilizamos para buscar informações sobre melhorias necessárias é a avaliação dos hospedes, como eles possuem um repertório de experiências de hospedagem em outros hotéis eles sugerem inúmeras melhorias, analisamos a viabilidade de algumas melhorias e por fim implantamos algumas. " (EH-5)

Estudos destacam que a Assimilação permite à organização, analisar, processar, interpretar e compreender as informações obtidas a partir das fontes externas (Gebauer et al., 2012; Szulanski, 1996; Zahra \& George, 2002). As práticas de interpretação e disseminação de conhecimentos oriundos de fontes externas, como por exemplo, reuniões interdepartamentais e parcerias com fornecedores, são relevantes para criação de novas oportunidades de negócios, identificados por Gebauer et al. (2012) e destacado na dimensão de Assimilação (PACAP), representada na Figura 2. As entrevistas sugerem que estas práticas são regulares nas organizações objetos deste estudo e justificadas nos relatos dos entrevistados:

“[...] no nosso hotel os gestores se reúnem quase que diariamente com suas equipes, reuniões ocorrem diariamente nos departamentos e para transmitir orientações $e$ compartilhar informações cada departamento utiliza um livro que é redigido a mão por cada turno de trabalho [...]" (EH-1)

"Então, nós somos pequenos então a gente tem essa facilidade, não precisa fazer muito documento, memorando, né? faz a reunião, a gente alinha tudo, faz a reunião setorial, e vai definindo as necessidades, o fluxo de informações geralmente funciona neste formato [...]" $(E H-2)$ 
"Com relação ao armazenamento a área de RH juntamente com a liderança de cada setor insere na descrição de cargos e no manual de procedimentos, isto possibilita que novos colaboradores saibam das práticas e rotinas para desempenhar sua função de forma eficaz $[\ldots] "(E H-3)$

"As reuniões são realizadas entre os líderes semanalmente na segunda feira pela manhã, participam todos os líderes e o gerente geral, nestas reuniões são discutidas as avaliações semanais dos hospedes, assim como outros assuntos pertinentes a operação do hotel." $(E H-3)$

A Capacidade Absortiva Realizada (RACAP) trata da capacidade da organização em Transformar (desenvolver e aprimorar as rotinas) e aplicar (executar e explorar) os conhecimentos externos (Zahra \& George, 2002). Na dimensão Transformação, o conhecimento externo é desenvolvido e aprimorado de acordo com as necessidades da organização. A Transformação é a capacidade que a empresa tem de recombinar e reconfigurar os conhecimentos existentes com àqueles adquiridos e assimilados pela organização de fontes externas (Denicolai et al., 2016; Gebauer et al., 2012; Zahra \& George, 2002). A Transformação resulta em rotinas que podem ser entendidas como Novas Práticas administrativas ou organizacionais que estão relacionadas às atividades diárias, de acordo com as normas culturais compartilhadas pela organização (Simcsik, 2001). Os entrevistados exemplificaram alguns conhecimentos externos adquiridos e assimilados, que foram Transformados em Novas Práticas:

"Com relação ao check-out expresso foi realizada uma análise de todas as avaliações dos hóspedes no ano de 2018, a nota com pior avaliação foi a agilidade no check-out, neste sentido nossa área de TI desenvolveu o sistema, me parece que buscaram informações técnicas em um hotel administrado pela marca nos Estados Unidos." (EH-1)

"[...] a gente trouxe uma inovação que é adaptação dos nossos cardápios a esta temática de vegano e vegetariano." (EH-2)

“[...] achamos interessante (ter uma loja na recepção e não um frigobar no quarto) $e$ montamos uma loja similar no hotel e retiramos os itens do frigobar, além de reduzir os custos com estornos o processo de check-out passou a ser realizado de forma mais ágil." $(E H-3)$

A dimensão Aplicação/Exploração, definida por Gebauer et al. (2012), tem seu foco na utilização do conhecimento externo transformado. Tal dimensão é fundamentada nas rotinas que permitem às organizações ampliarem e alavancarem as capacidades existentes, ou construir novas, baseadas na incorporação do conhecimento externo adquirido, assimilado e transformado em Novas Práticas (Zahra \& George, 2002). Essas rotinas oferecem mecanismos estruturais, sistêmicos e processuais que capacitam as organizações a conquistar vantagens 
competitivas sustentáveis (Gebauer et al., 2012; Salunke et al., 2019; Zahra \& George, 2002). Neste estudo foram identificadas algumas práticas aplicadas à rotina do hotel que contribuíram para melhoria do negócio, conforme relato de alguns entrevistados:

"[...] a inovação foi implantada no ano passado (2019), modificamos o sistema de reservas. [...] implantamos um channel manager que gerencia as tarifas e disponibilidades de apartamentos [...] isso resultou no aumento da ocupação do empreendimento e na redução de overbooking, sem contar que diminuiu a sobrecarga de trabalho dos colaboradores que atuam na área de comercial. (EH-3)

"[...] implantamos um sistema nas duas piscinas do hotel de geradores de cloro eletrônicos, isto possibilitou que as piscinas tivessem sempre tratadas e reduziu nosso consumo de cloro e de outros produtos químicos que eram necessários para o tratamento da água [...]” (EH-1)

As inovações oriundas da capacidade de absorção da organização do setor de hospitalidade, exercem um papel fundamental para competitividade e o sucesso das empresas do ramo (Binder, 2019; Enz \& Way, 2016). Tais organizações requerem melhorias constantes em serviços ou produtos, levando em consideração o papel fundamental dos funcionários, principalmente, considerando dois fatores: i. funcionários que interajam com clientes; e ii. disposição destes funcionários em promover melhorias nos serviços (Chang et al., 2018), ou seja, as inovações neste ramo ocorrem por meio de uma intensa relação entre funcionários da linha de frente e clientes que interagem e estão simultaneamente envolvidos no processo de cocriarão de valor (Cadwallader et al., 2010; Chang et al, 2018).

“[...] um hóspede escreveu em sua avaliação que seria interessante disponibilizar ao menos duas opções de pratos veganos, como nosso chef de cozinha não era especialista neste tipo de cozinha o hotel pagou um curso para ele. Hoje existem diversas opções novas de pratos veganos no nosso cardápio. (EH-1)

"[...] tem a haver com esta parte de reservas. A gente fez toda uma reestruturação no departamento de reserva no sentido de implantar motor de reserva para reservas diretas [...] então a gente adquiriu, fez toda uma reestruturação aqui na parte comercial do hotel, e a gente buscou esta melhoria. [...] Além do que o próprio motor também puxa muita hospedagem pra uma negociação direta aqui nas reservas [...] (EH-4)

As inovações na indústria hoteleira normalmente ocorrem por meio de inovações incrementais - predominantemente captadas no ambiente externo - e são ocasionadas, em sua maioria, quando a organização enfrenta situações adversas, como por exemplo, crises, redução de faturamento e perda do mercado para concorrentes ou mesmo uma pandemia (Hon \& Lui, 2016). Apesar do foco deste estudo não ter sido a atual situação que o mundo enfrenta de pandemia e isolamento social, dada a especificidade e os impactos causados pela paralização 
das atividades econômicas em virtude da Covid-19, os respondentes relataram algumas práticas adotadas para atender os novos protocolos de segurança:

“[...] neste primeiro momento fazer um investimento em material de segurança, EPI, material de controle, materiais de higienização, tivemos que seguir todo este protocolo, treinar equipe e tudo pra seguir com isso. Então houve um investimento grande neste período. Nós não estávamos preparados pra isso." (EH-1)

"A AB (alimentos e bebidas) mudou bastante, porque a gente está fazendo agora tudo à la carte, já tínhamos o buffet. Aqui é tudo pensão completa, então a gente já é bem conhecido no mercado pela gastronomia que a gente tem aqui, uma gastronomia muito boa. À noite a gente já tinha um à la carte muito bom e a gente transformou o dia em à la carte e o café da manhã a gente teve que se reinventar também. Praticamente tudo à la carte agora." $(E H-2)$

"Tivemos que trabalhar a essência de móveis rústicos, através das mesas com madeira crua. Isso é uma coisa que também foi implantada. A gente eliminou toda a parte de toalhas de hotel [...] a gente está trabalhando tudo com a mesa de madeira crua, rústica nos restaurantes e nas áreas de coffee break." (EH-3)

"Porque quando você tem que seguir estes protocolos, aí cada departamento tem a sua especificidade cada um, né? Desde o momento que chega na recepção já tem todo um protocolo para seguir a higienização das unidades mudou tudo também, o protocolo." $(\mathrm{EH}-4)$

"No início foi complicado, mas à medida que fomos treinando as equipes, os protocolos de segurança foram sendo incorporados naturalmente, a principal inovação foi que tivemos que mudar o sistema de buffet self service para a la carte e o café da manhã passamos a servir no quarto do hospede, fomos nos adaptando." (EH-5)

Os cinco respondentes nas entrevistas mencionaram que os processos de aquisição de conhecimentos externos dos empreendimentos ocorrem frequentemente (de Lima et al., 2021). Além disso, "Inovação" foi outro termo que está em evidência e foi muito citado pelos entrevistados, pois é o resultado dos processos da capacidade absortiva potencial (PACAP) e realizada (RACAP) (Binder, 2019; Enz \& Way, 2016).

\section{Conclusões}

O objetivo do estudo foi identificar quais são as práticas existentes para captar e absorver conhecimentos externos em empreendimentos hoteleiros. Nesse sentido, o presente estudo corrobora com o modelo proposto por Zahra e George (2002), uma vez que os achados da pesquisa constataram, por meio das entrevistas realizadas, que os hotéis possuem práticas de aquisição de conhecimentos externos similares, como por exemplo, participação em feiras e eventos do setor, análise das avaliações e sugestões dos hóspedes. Porém, conhecimentos 
prévios de mercado foi citado por apenas um dos respondentes como forma predominante de implementação de melhorias nos serviços prestados, apesar de não ser esperado, a resposta deste entrevistado é coerente, pois a contratação de novos colaboradores possibilita que a organização aumente seu repertório de conhecimentos de mercado e, consequentemente, remodele as práticas existentes visando promover vantagem competitiva sustentável.

A prática de aquisição predominante e compreendida como a mais importante pelos respondentes são as melhorias geradas por meio da análise das avaliações e sugestões citadas pelos hóspedes, reforçando a sustentação teórica que afirma que a interação de funcionários e clientes é fundamental para que melhorias sejam implementadas no serviço hoteleiro, fomentando a cocriação de valor (Enz \& Way, 2016).

Com relação ao processo de assimilação, todos os respondentes relataram que as reuniões de equipe são fundamentais para disseminar e absorver os conhecimentos externos. Os respondentes mencionaram que as reuniões têm este caráter fundamental, pois a estrutura de um empreendimento hoteleiro não é tão burocratizada ou sistematizada quanto outros segmentos de mercado. Apenas um dos respondentes, que atua em um hotel de porte médio, mencionou que após reuniões regulares são inseridas nos manuais de procedimentos e na descrição de cargos as novas práticas a serem adotadas. Assim, os achados da pesquisa relacionados às práticas convergentes e divergentes nos processos de aquisição, assimilação, transformação e aplicação dos conhecimentos possibilitaram afirmar que o objetivo deste estudo foi alcançado.

Este estudo contribui para o desenvolvimento do campo teórico sobre capacidade absortiva do setor hoteleiro, preenchendo uma importante lacuna na literatura que apresenta escassez de estudos que investigaram as quatro dimensões referentes às práticas de capacidades absortivas potencial (PACAP) e realizada (RACAP), conforme apresentado por Zahra e George (2002). Como contribuição gerencial, o estudo fornece um guia para os gestores, das principais práticas identificadas e em como desenvolver estes processos de forma eficaz e consequentemente, promover inovações nos serviços prestados.

O estudo apresenta importantes contribuições teóricas e gerenciais, contudo, apresenta algumas limitações. Dentre elas a generalização dos achados em virtude do tamanho da amostra. Sugere-se que estudos futuros possam ser realizados com pesquisas quantitativas, permitindo generalização ou mesmo com outras fontes de coleta de dados, por exemplo, observação participante ou não participante ou mesmo grupo focal. Outrossim sugere-se que a capacidade absortiva seja explorada em futuras pesquisas como "fonte de" ou "incentivo à" 
cocriação de valor; ou ainda explorar por outro ângulo, como por exemplo, de que maneira a cocriação de valor influência na capacidade absortiva, visto que pesquisas indicam que quando há cocriação de valor, gatilhos cognitivos e/ou afetivos, despertam nos turistas um desejo de compartilhar suas experiências com outras pessoas (Ribeiro et al., 2021), e os entrevistados da presente pesquisa revelaram que a opinião dos hóspedes é uma das maiores fontes de capacidade absortiva. Estas sugestões poderão resultar em maiores levantamentos e contribuições práticas para a área.

\section{Agradecimentos}

"O presente trabalho foi realizado com apoio da Coordenação de Aperfeiçoamento de Pessoal de Nível Superior - Brasil (CAPES) - Código de Financiamento 001 e do Conselho Nacional do Desenvolvimento Científico e Tecnológico (CNPq)".

\section{Referências}

Bardin, L. (2016). Análise de conteúdo (Edição revista e actualizada) (1a.). Edições 70.

Benbasat, I., Goldstein, D. K., \& Mead, M. (1987). The case research strategy in studies of information systems. MIS quarterly, 369-386. https://doi.org/10.2307/248684

Bessant, J., Von Stamm, B., Moeslein, K. M., \& Neyer, A.-K. (2010). Backing outsiders: Selection strategies for discontinuous innovation. $R \& d$ Management, 40(4), 345356. https://doi.org/10.1111/j.1467-9310.2010.00606.x

Binder, P. (2019). A network perspective on organizational learning research in tourism and hospitality. International Journal of Contemporary Hospitality Management. https://doi.org/10.1108/IJCHM-04-2017-0240

Bontis, N., Janošević, S., \& Dženopoljac, V. (2015). Intellectual capital in Serbia's hotel industry. International Journal of Contemporary Hospitality Management. https://doi.org/10.1108/IJCHM-12-2013-0541

Cadwallader, S., Jarvis, C. B., Bitner, M. J., \& Ostrom, A. L. (2010). Frontline employee motivation to participate in service innovation implementation. Journal of the Academy of Marketing Science, 38(2), 219-239. https://doi.org/10.1007/s11747-0090151-3

Chalkiti, K. (2012). Knowledge sharing in dynamic labour environments: Insights from Australia. International Journal of Contemporary Hospitality Management. https://doi.org/10.1108/09596111211226806 
Chang, S., Gong, Y., \& Shum, C. (2011). Promoting innovation in hospitality companies through human resource management practices. International Journal of Hospitality Management, 30(4), 812-818. https://doi.org/10.1016/j.ijhm.2011.01.001

Chang, Y.-Y., Chao, W.-C., Chang, C.-Y., \& Chi, H.-R. (2018). Transformational leadership influence on unit performance: Cross-level moderated mediation evidence. Leadership \& Organization Development Journal, 39(4), 554-571. https://doi.org/10.1108/LODJ08-2017-0224

Christensen, C. M. (1997). The Innovator's Dilemma. Harvard Business School Press. Boston, MA.

Cohen, W. M., \& Levinthal, D. A. (1990). Absorptive capacity: A new perspective on learning and innovation. Administrative science quarterly, 128-152. https://doi.org/10.2307/2393553

Creswell, J. W. (2010). Projeto de pesquisa métodos qualitativo, quantitativo e misto. In Projeto de pesquisa métodos qualitativo, quantitativo e misto (3 ed., p. 296p.). Artmed.

Cyert, R. M., \& March, J. G. (1963). A behavioral theory of the firm. Englewood Cliffs, NJ, 2(4), 169-187.

de Lima, A. A., Costa, B. K., Shinohara, E. E., \& da Silva Bezerra, C. M. (2021). Estudo da Evolução Conceitual-Teórica da Capacidade Absortiva no Campo da Hotelaria. Marketing \& Tourism Review, 6(1). https://doi.org/10.29149/mtr.v6i1.6530

Deakin, H., \& Wakefield, K. (2014). Skype interviewing: Reflections of two PhD researchers. Qualitative research, 14(5), 603-616. https://doi.org/10.1177/1468794113488126

Denicolai, S., Ramirez, M., \& Tidd, J. (2016). Overcoming the false dichotomy between internal R\&D and external knowledge acquisition: Absorptive capacity dynamics over time. Technological Forecasting and Social Change, 104, 57-65. https://doi.org/10.1016/j.techfore.2015.11.025

Dzhengiz, T., \& Niesten, E. (2019). Competences for environmental sustainability: A systematic review on the impact of absorptive capacity and capabilities. Journal of Business Ethics, 1-26. https://doi.org/10.1007/s10551-019-04360-z

Eisenhardt, K. M. (1989). Building Theories from Case Study Research. Academy of Management Review, 14(4), 532-550. https://doi.org/10.5465/amr.1989.4308385

Enz, C. A., \& Way, S. A. (2016). Implementing service innovations in European hotels. Service science, $8(2), 97-107$. https://doi.org/10.1287/serv.2015.0121

Flick, U. (2020). Triangulation. In G. Mey \& K. Mruck (Orgs.), Handbuch Qualitative Forschung in der Psychologie: Band 2: Designs und Verfahren (p. 185-199). Springer Fachmedien. https://doi.org/10.1007/978-3-658-26887-9_23 
Flor, M. L., Cooper, S. Y., \& Oltra, M. J. (2018). External knowledge search, absorptive capacity and radical innovation in high-technology firms. European Management Journal, 36(2), 183-194. https://doi.org/10.1016/j.emj.2017.08.003

Flores, J. G. (1994). Análisis de datos cualitativos: Aplicaciones a la investigación educativa [PhD Thesis]. Universidad de Sevilla, Facultad de Filosofía y Ciencias de la Educación.

Gebauer, H., Worch, H., \& Truffer, B. (2012). Absorptive capacity, learning processes and combinative capabilities as determinants of strategic innovation. European Management Journal, 30(1), 57-73. https://doi.org/10.1016/j.emj.2011.10.004

Golafshani, N. (2003). Understanding reliability and validity in qualitative research. The qualitative report, 8(4), 597-607.

Khale, P. J., Haass, C., Kretzschamr, H. A., \& Neumann, M. (2002). Structure/function of alpha-synuclein in health and disease: Rationl development of animal models for Parkinson's and related disease. J. Neurochem, 82, 449457. https://doi.org/10.1046/j.1471-4159.2002.01020.x

Hallin, C. A., \& Marnburg, E. (2008). Knowledge management in the hospitality industry: A review of empirical research. Tourism management, 29(2), 366-381. https://doi.org/10.1016/j.tourman.2007.02.019

Herriot, P., Chalmers, C., \& Wingrove, J. (1985). Group decision making in an assessment centre. Journal of Occupational Psychology, 58(4), 309312. https://doi.org/10.1111/j.2044-8325.1985.tb00203.x

Hon, A. H., \& Lui, S. S. (2016). Employee creativity and innovation in organizations. International Journal of Contemporary Hospitality Management. https://doi.org/10.1108/IJCHM-09-2014-0454

Kim, T., \& Lee, G. (2012). A modified and extended Triandis model for the enablersprocess-outcomes relationship in hotel employees' knowledge sharing. The service industries journal, 32(13), 2059-2090. https://doi.org/10.1080/02642069.2011.574276

Lane, P. J., Koka, B. R., \& Pathak, S. (2006). The reification of absorptive capacity: A critical review and rejuvenation of the construct. Academy of management review, 31(4), 833863. https://doi.org/10.5465/amr.2006.22527456

Lev, S., Fiegenbaum, A., \& Shoham, A. (2009). Managing absorptive capacity stocks to improve performance: Empirical evidence from the turbulent environment of Israeli hospitals. European Management Journal, 27(1), 13-25. https://doi.org/10.1016/j.emj.2008.04.001

Lewin, A. Y., Massini, S., \& Peeters, C. (2011). Microfoundations of internal and external absorptive capacity routines. Organization science, 22(1), 81-98. https://doi.org/10.1287/orsc.1100.0525 
Loureiro, R., Ferreira, J. J., \& Simões, J. M. M. (2019). Learning Dynamic Capabilities in healthcare organizations-a qualitative research. Revista de Gestão e Sistemas de Saúde-RGSS, 8(3), 283-296. https://doi.org/10.5585/RGSS.v8i3.11533

Lowik, S., van Rossum, D., Kraaijenbrink, J., \& Groen, A. (2012). Strong ties as sources of new knowledge: How small firms innovate through bridging capabilities. Journal of Small Business Management, 50(2), 239-256. https://doi.org/10.1111/j.1540627X.2012.00352.X

Moorman, C., \& Miner, A. S. (1998). The convergence of planning and execution: Improvisation in new product development. Journal of marketing, 62(3), 1-20. https://doi.org/10.1177/002224299806200301

Mowery, D. C., \& Oxley, J. E. (1995). Inward technology transfer and competitiveness: The role of national innovation systems. Cambridge journal of economics, 19(1), 67-93. https://doi.org/10.1093/oxfordjournals.cje.a035310

Nelson, R. R., \& Winter, S. G. (2009). An evolutionary theory of economic change. harvard university press.

Nieves, J., \& Diaz-Meneses, G. (2018). Knowledge sources and innovation in the hotel industry. International Journal of Contemporary Hospitality Management. https://doi.org/10.1108/IJCHM-07-2016-0341

Noerchoidah, N., \& Harjanti, D. (2019). Exploring the relationship between procedural justice and innovative work behavior in hospitality industry. Jurnal Manajemen dan Kewirausahaan, 21(1), 21-31. https://doi.org/10.9744/jmk.21.1.21-31

Nonaka, I., \& Takeuchi, H. (1997). Criação de conhecimento na empresa. Elsevier Brasil.

Parmigiani, A., \& Howard-Grenville, J. (2011). Routines revisited: Exploring the capabilities and practice perspectives. Academy of Management annals, 5(1), 413-453. https://doi.org/10.5465/19416520.2011.589143

Pattanasing, K., Aujirapongpan, S., \& Srimai, S. (2019). Dynamic capabilities and high performance organization of hotel business: Empirical investigation into world class tourism destination. Tourism and hospitality management, 25(2), 377-401. https://doi.org/10.20867/thm.25.2.8

Pisano, G. P. (2017). Toward a prescriptive theory of dynamic capabilities: Connecting strategic choice, learning, and competition. Industrial and Corporate Change, 26(5), 747-762. https://doi.org/10.1093/icc/dtx026

Polanyi, M. (1966). The logic of tacit inference. Philosophy, 41(155), 1-18. https://doi.org/10.1093/icc/dtx026

Ribeiro, T. de L. S., Costa, B. K., \& Freire, O. B. D. L. (2021). Cocriação de Valor no Turismo-Validação e Replicação de Escala em Relação à Intenção de Recomendação Boca-a-Boca. Revista Brasileira de Pesquisa em Turismo, 15. https://doi.org/10.7784/rbtur.v15i2.1924 
Richardson, R. J. (2017). Pós-Graduação-Metodologia-Pesquisa Social: Métodos e TécnicasMétodos Quantitativos e Qualitativos-Capitulo 5. Editora ATLAS SA.

Sainaghi, R., Phillips, P., \& Zavarrone, E. (2017). Performance measurement in tourism firms: A content analytical meta-approach. Tourism Management, 59, 36-56. https://doi.org/10.1016/j.tourman.2016.07.002

Salunke, S., Weerawardena, J., \& McColl-Kennedy, J. R. (2019). The central role of knowledge integration capability in service innovation-based competitive strategy. Industrial Marketing Management, 76, 144-156. https://doi.org/10.1016/j.indmarman.2018.07.004

Simcsik, T. (2001). OSM: Organizações, sistemas e métodos. Futura.

Szulanski, G. (1996). Exploring internal stickiness: Impediments to the transfer of best practice within the firm. Strategic management journal, 17(S2), 27-43. https://doi.org/10.1002/smj.4250171105

Tatto, L., \& Bordin, R. A. (2016). Filosofia e Gestão do Conhecimento: Um estudo do conhecimento na perspectiva de Nonaka e Takeuchi. Cadernos EBAPE. BR, 14(2), 340-350. https://doi.org/10.1590/1679-395141463

Teece, D. J. (2007). Explicating dynamic capabilities: The nature and microfoundations of (sustainable) enterprise performance. Strategic management journal, 28(13), 13191350. https://doi.org/10.1002/smj.640

Thomas, R., \& Wood, E. (2014). Innovation in tourism: Re-conceptualising and measuring the absorptive capacity of the hotel sector. Tourism Management, 45, 39-48. https://doi.org/10.1016/j.tourman.2014.03.012

Tsai, W. (2001). Knowledge transfer in intraorganizational networks: Effects of network position and absorptive capacity on business unit innovation and performance. Academy of management journal, 44(5), 996-1004. https://doi.org/10.5465/3069443

Tuzzo, S. A., \& Braga, C. F. (2016). O processo de triangulação da pesquisa qualitativa: O metafenômeno como gênese. Revista Pesquisa Qualitativa, 4(5), 140-158. https://editora.sepq.org.br/rpq/article/view/38

Vega-Jurado, J., Gutiérrez-Gracia, A., Fernández-de-Lucio, I., \& Manjarrés-Henríquez, L. (2008). The effect of external and internal factors on firms' product innovation. Research policy, 37(4), 616-632. https://doi.org/10.1016/j.respol.2008.01.001

Vergara, S. C. (2013). Projetos e relatórios de pesquisa em administração (14 ed.). Atlas. Walsh, J. P., \& Ungson, G. R. (1991). Organizational memory. Academy of management review, 16(1), 57-91. https://doi.org/10.5465/amr.1991.4278992

Winter, S. G. (2000). The satisficing principle in capability learning. Strategic management journal, 21(10-11), 981-996. https://doi.org/10.1002/10970266(200010/11)21:10/11<981::AID-SMJ125>3.0.CO;2-4 
Bezerra, C. M. S., Ramos, H. R., Lima, A. A., \& Shinohara, E. E. (2022, jan./abr.). Capacidade absortiva no contexto da indústria hoteleira: uma análise de práticas de absorção de conhecimentos

Woods, M., Paulus, T., Atkins, D. P., \& Macklin, R. (2016). Advancing qualitative research using qualitative data analysis software (QDAS)? Reviewing potential versus practice in published studies using ATLAS. ti and NVivo, 1994-2013. Social Science Computer Review, 34(5), 597-617. https://doi.org/10.1177/0894439315596311

Zahra, S. A., \& George, G. (2002). Absorptive capacity: A review, reconceptualization, and extension. Academy of management review, 27(2), 185-203. https://doi.org/10.5465/amr.2002.6587995

Zahra, S. A., Sapienza, H. J., \& Davidsson, P. (2006). Entrepreneurship and dynamic capabilities: A review, model and research agenda. Journal of Management studies, 43(4), 917-955. https://doi.org/10.1111/j.1467-6486.2006.00616.x

Zhang, H., Gupta, S., Sun, W., \& Zou, Y. (2019). How social-media-enabled co-creation between customers and the firm drives business value? The perspective of organizational learning and social Capital. Information \& Management, 103200. https://doi.org/10.1016/j.im.2019.103200

Zollo, M., \& Winter, S. G. (2002). Deliberate learning and the evolution of dynamic capabilities. Organization science, 13(3), 339-351.

https://doi.org/10.1287/orsc.13.3.339.2780 